\title{
DIETORECS: Travel Advisory for Multiple Decision Styles
}

\author{
Daniel R. Fesenmaier ${ }^{a}$,Francesco Ricci ${ }^{b}$ Erwin Schaumlechner ${ }^{\mathrm{c}}$ Karl Wöber ${ }^{\mathrm{d}}$, and \\ Cristiano Zanella ${ }^{\mathrm{e}}$ \\ ${ }^{a}$ National Laboratory for Tourism and eCommerce \\ University of Illinois at Urbana Champaign, USA \\ drfez@uic.edu \\ ${ }^{\mathrm{b}}$ Electronic Commerce and Tourism Research Laboratory \\ ITC-irst, Italy \\ ricci@itc.it \\ ${ }^{\mathrm{c}}$ Tiscover AG - Travel Information Systems \\ Softwarepark Hagenberg, Austria \\ erwin.schaumlechner@tiscover.com \\ ${ }^{\mathrm{d}}$ Institute for Tourism and Leisure Studies \\ Vienna University of Economics and Business Administration, Austria \\ woeber@isis.wu-wien.ac.at \\ ${ }^{\mathrm{e}}$ Azienda Promozione Turistica del Trentino,Italy
}

\begin{abstract}
This paper presents Dietorecs, a novel case-based travel planning recommender system. Dietorecs has been designed by incorporating a human decision model that stresses individual differences in decision styles. Dietorecs supports decision styles by means of an adaptive behavior which is learned exploiting a case base of recommendation sessions that are stored by the systems. Users can enter the system through three main functional doors that fit groups of decision styles, but they can eventually switch the type of support required. The dialogue (questions) is personalized using both the user model (cases) and statistics over the data available in the virtual catalogues provided by two DMOs.
\end{abstract}

Keywords: recommender systems; travel planning; decision modeling, personalization.

\section{Introduction}

There is a growing number of web sites that support a traveller in the selection of travel destinations or travel products (e.g., flight or hotel). Typically, the user is 
required to input product constraints or preferences that are matched by the system in an electronic catalogue. Major eCommerce web sites dedicated to tourism such as Expedia, Travelocity, and Tiscover have started to cope with travel planning by incorporating recommender systems, i.e., applications that provide advice to users about products (Schafer et al., 2001). Recommender systems for travel planning try to mimic the interactivity observed in traditional counselling sessions with travel agents (Delgado and Davidson, 2002). The current generation of travel recommender systems focus on destination selection and do not support the user through a personalized interaction in bundling a tailor-made trip made of one or more locations to visit, an accommodation and additional attractions (museum, theatre, etc.).

The Dietorecs ${ }^{1}$ system extends current recommender systems by incorporating a human choice model extracted from both the literature and the empirical analysis of the traveller's behaviour. Dietorecs supports the selection of travel products (e.g., a hotel or a visit to a museum or a climbing school) and building a 'travel bag'; that is, a coherent (from the user point of view) bundling of products. Dietorecs also supports multiple decision styles by letting the user 'enter' the system through three main 'doors': iterative single item selection, complete travel selection and inspiration driven selection. The first door enables the most experienced user to efficiently navigate in the potentially overwhelming information provided by the two integrated data bases (Tiscover and APT Trentino). The user is allowed to select whatever products he/she likes and in the preferred order, using the selections done up to a certain point (and in the past) to personalize the next stage. The second door enables the user to select a personalized trip that bundles together items available in the catalogue. The personalized plan is constructed by "reusing" the structure and main content of trips either built by other users or available from some provider. The third door allows an inspiration seeking user to choose a complete trip by exploiting a simpler user interface (icon based) as well an interaction which is kept at the minimum length as possible. It must be stressed that all these decision styles are supported in a uniform and seamless way by means of a graphical user interface. Hence, switching from one style to another is always possible and easy to do.

The next two sections describe our conceptual approach to travel planning and illustrate how the notion of decision styles emerged from the research. Then, we briefly describe the fundamental element of the designed application and its technological implementation. We end the paper by summarizing the state of the work of this research project.

\footnotetext{
${ }^{1}$ This work has been partially funded by the European Union's Fifth RTD Framework Programme (under contract DIETORECS IST-2000-29474).
} 


\section{Conceptual Approaches for Solving the Application Problem}

An in-depth understanding of the destination choice process, those factors considered during travel planning, and the interaction between human and computer mediated environments are an important foundation for any destination recommendation system (Hwang, Gretzel and Fesenmaier 2002). Hence, developers of a travel advisory system cannot focus solely on computer science theory but must also consider the findings and achievements in travel decision theory. In tourism research, travel decision theory is one of the most comprehensively investigated areas with a relatively long tradition (see Mayo and Jarvis 1981 for one of the first textbooks devoted to the psychology of leisure travel). Many conceptual approaches to understanding travel decision making have been proposed. In general, these approaches can be conceptualised into four different frameworks: (1) Choice set models (e.g. Crompton and Ankomah 1993; Um and Crompton 1990); (2) General travel models (e.g. Woodside and Lysonski 1989); (3) Decision net models (Fesenmaier and Jeng 2000); and, (4) Multi-destination travel models (e.g. Lue, Crompton and Fesenmaier 1993). Accumulated literatures on travel destination choice indicate that the variables used to explain and predict one's destination choice can be classified into two broad categories: (1) personal and (2) travel characteristics. Personal characteristics encompass socio-economic characteristics as well as one's psychological/cognitive traits. Travel characteristics include situational factors that make the travel distinguishable from other travel. Although there exists a rich literature explaining each of these conceptual approaches, only a few contributions focus attention on those factors that need to be integrated into human-centric destination recommendation systems. Another important limitation concerning the applicability of travel decision theory relates to its demand-driven focus, hence requesting a universal supplier database. The majority of models are based upon indepth observational studies investigating the needs and/or benefits of consumers and largely ignore the characteristics and constraints associated with a specific media. Traditionally, research initiatives have focused on what is 'ideal' but not on what is 'optimal' within a certain decision making environment. It is, therefore, not surprising that IT developers and an increasing number of tourism researchers who are involved in building intelligent travel advisory systems question the practical value of many of the traditional (purely conceptual) models.

\section{Travel Decision Styles}

Decomposition of a behavioural framework linking destination choice, information search and human-computer interaction enables one to develop a set of propositions that guide the design of a travel destination recommendation system (Hwang, Gretzel 
and Fesenmaier 2002): (1) Destinations recommended should vary according to the nature of each trip and user characteristics; (2) Recommendations should reflect personal differences in the nature of the information sought and processing styles; and, (3) The destination recommendation system should facilitate experiential aspects of the decision and information searching process. In order to efficiently match travellers' needs with the offers in the databases a travel destination recommendation system should adapt itself to the user in terms of: (1) Hierarchy of questions (sequence of sub-decisions); (2) Mode of representation (information search vs. experiential); and, (3) Degree of recommendation vs. search functions (user or system driven sessions).

With these objectives in mind, it becomes clear that the dynamics of decision-making and information retrieval processes need to be examined. Therefore, an observational study in three design variations was conducted in order to investigate the individual trip planning and information behaviour process from a dynamic point of view (Grabler and Zins 2002). The exploratory approach resulted in six different decision styles which were suggested to be useful when setting up a new generation ITsupported travel recommendation system: (1) Highly pre-defined users; (2) Accommodation-oriented users; (3) Recommendation-oriented users; (4) Geographyoriented users; (5) Price-oriented users; and, (6) The individual traveller. The decision styles link the various issues raised in the consumer behaviour literature with an operational perspective in order to integrate the key factors (socioeconomic, psychological/cognitive variables and trip characteristics) into a travel recommendation system.

Ideally, the system must enable a (new) user entering the travel destination recommendation system to be classified quickly in order to provide him/her the optimal navigational path and mode of presentation (type and sequence of questions, graphical widgets selection, length of interaction etc.). The user should be able to influence the dialogue management component by explicitly volunteering information useful to determine his/her decision style. This can be achieved by self selection of the decision style, for instance, by presenting iconic descriptions of the styles. Although the study by Grabler and Zins (2002) recommends that information on a user's decision style should be acquired at a very early stage in the session, the system should provide the possibility of switching between different interface styles. Again, this can be achieved by self-selection of the user or derived from a pattern of user interaction with the computer. Information presented at a later stage of the user session should be structured differently according to the requirements of the respective decision styles. However, the following three stages are used to decompose the dialogue: 
(1) Filtering: The user must be able to enter the primary variables/constraints that describe his/her decision style; however, not all information categories are required at the beginning of the dialogue.

(2) Specification: Additional information related to the responses provided in first stage are presented to the user. An important aspect of this stage is that the user believes that only personally 'important' features are asked.

(3) Selection/sorting: The user must be able to make his/her final decision based on a short number of alternatives presented. This list may be sorted by one (or more) of the key factors associated with the identified decision style and/or specified during the decision process by the user.

As the number of possible offers should be counted and provided to the user at any time, it is possible that a specific user session does not require all three stages as the number of matched offers may be limited. Additional information which appears to be important in describing the characteristics of the decision process and therefore needs to be monitored and stored during a session include: Usage type, sequence of sub-decisions, flexibility/rigidity of trip characteristics, degree of pre-specification, number of alternatives, decision style, and experiential proneness.

\section{The proposed Approach}

The theoretical considerations provided above sets the requirements for a travel planning recommender system that must support, by means of an adaptive behaviour, rather different decision styles and must personalize the suggestions on the base of both personal and travel characteristics. In this section we shall describe the basic design choices of the Dietorecs recommender system and illustrate a typical $\mathrm{man} /$ machine interaction. The Dietorecs system is based on the following elements:

- Bundling a mix-and-match travel. Dietorecs basically supports the user in building a personalized travel plan that can either comprise a pre-packaged offer or can be obtained by iteratively selecting travel components (items) such as locations to visit, accommodations, attractions, services. Item selection dialogue is driven by the personal and travel characteristics that are structurally decomposed into what are referred to as "general" and "detailed travel wishes".

- Allow the user to enter through three functional doors. The user can build (configure) his travel plan by means of three top system functions that acts as different doors to enter the system. These doors enable users to provide in whatever order and amount he/she likes general and detailed travel wishes. The first door allows the user to select whatever products he likes and in the preferred order. The second door enables the user to select a personalized trip that bundles 
together items available in the catalogues. The third door enables an inspiration seeking user to choose a complete trip exploiting (selecting and modifying) examples of travels shown by means of a user interface which is strongly based on images and that minimizes the interaction length.

- Decision styles and functional doors. Decision styles are initially mapped (probabilistically) to these functional doors. This means that Dietorecs is bootstrapped with a default assignment of decision styles to doors (for instance the "highly predefined user" to the first door), but user activity logs, stored as cases, will provide data for training the Dietorecs classifier to (1) identify the decision style (2) suggesting the user to switch to another door.

- Register the user interaction session as a case. The adaptive behaviour of Dietorecs is based on a structured representation of the interaction session that is stored as a case in case-based reasoning system (Ricci et al, 2002a). A case includes general and specific travel wishes acquired during the interaction, items included in the 'travel bag', feed-back provided by the user on the items selected, ordered list of the system functions called during the interaction (activity log).

- Personalise the questions posed to the user using cases and catalogue analysis. After having acquired some travel wishes from the user, Dietorecs poses in-context questions that either try to further specify the travel wishes, whose effect is to tighten the search, either to relax conditions that cannot be satisfied. The identification of those travel wishes that could be asked or should be relaxed rely on: (1) the analysis of the users behaviours stored in the cases (statistics over user explicit wishes), (2) constraint relaxation techniques, and (3) information theory indicators, such entropy, computed on the catalogues of products (Ricci et al., 2002b).

- Personalise the recommendations using collaboration filtering through case similarity. The items (and the complete trips)) suggested by Dietorecs are ranked according to a collaboration-via-content based approach (Pazzani, 1999). In fact, item filtered according to the user travel wishes are then sorted such that those contained (or more similar to) in similar recommendation sessions (cases) are scored best. With this respect Dietorecs is a hybrid recommender system that overcomes classical problems of pure collaborative-based approaches such as huge amounts of registered user logs data needed to deliver recommendations.

In the rest of this section we provide a sample session with Dietorecs. For lack of space we cannot cover all the potential interactions, initiated by entering into the three doors mentioned above.

When starting to use the system the tourist must first decide how to search through the travel cases managed by the system. If the tourist is more recommendation-driven, then the selection process will be supported from the system in the form of pictorial 
representations of former cases (third door). The user can qualify the suggestions by labelling them as interesting versus not interesting, which should result in a case near enough to the user's travel imaginations to be selected. Otherwise, information-driven tourists with more concrete ideas such as travel destination or accommodation category normally prefer a more straightforward access to the travel cases as shown in Fig. 1 (first door).

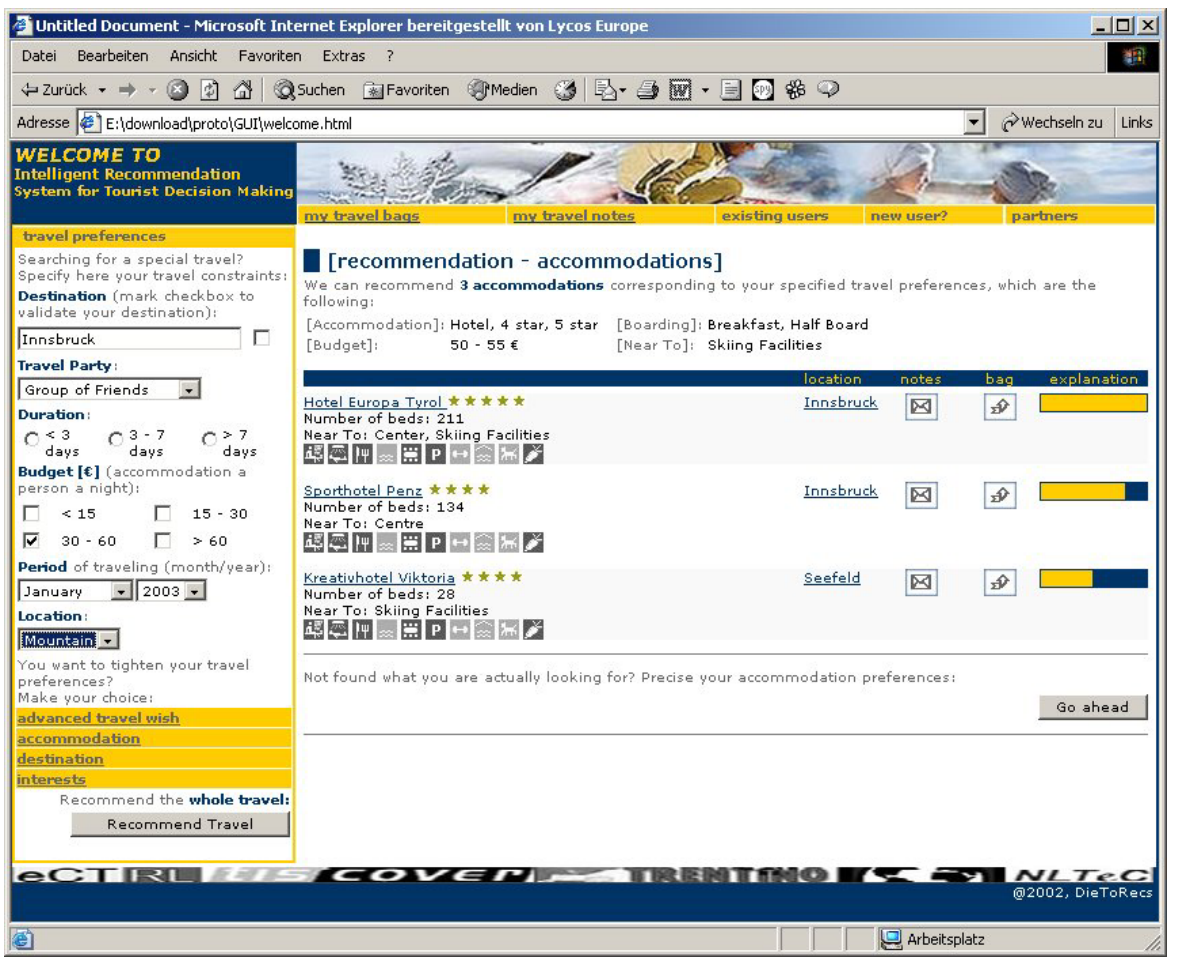

Fig. 1. Dietorecs Application Sketch - Item Search

The left frame contains all travel preferences, clustered into general travel preferences and so-called item preferences, represented by accommodation, destination and interest items. These are the elementary components of a case, namely of the 'travel bag'. Based on these preferences it is up to the system to support the user with suitable recommendations. After retrieving relevant cases from the case base and comparing their content with real items contained in the virtual catalogues of an integrated tourist information system, Dietorecs offers the user a list of recommendations. The recommendations are sorted according to the degree of similarity between found items, satisfying travel preferences, and items contained in 
past cases. If a user decides that one of the listed items fits, he/she can add it to his/her current travel bag by clicking the button in the column "to bag". A travel bag collects the selections made by the user from different item types and can be stored by assigning a label. Users of the system if they are registered can manage as many travel bags as supported by the system.

Advanced functionalities include the possibility to complete an already created travel bag by finding the most similar case or the chance to find similar items from within the current travel bag and allow a comfortable and flexible creation of suitable travel recommendations.

\section{Technical Solutions and Software Architecture}

The system exploits a case base of travel bags that is built by the community of Dietorecs users as well as catalogues provided by Tiscover AG and APT Trentino (DMO). The proposed case structure is hierarchical (Ricci et al., 2002b) and implemented as an XML view over a relational data base. Dietorecs integrates casebased reasoning with interactive query management. When asked to retrieve a travel product, Dietorecs tries to cope strictly with user needs and, if this is not possible, it suggests query changes that will produce acceptable results. Similarity-based retrieval is exploited: (1) when a complete travel is searched (second and third door) and (2) when the single products (as the result set of the user's query) must be ranked (first door).

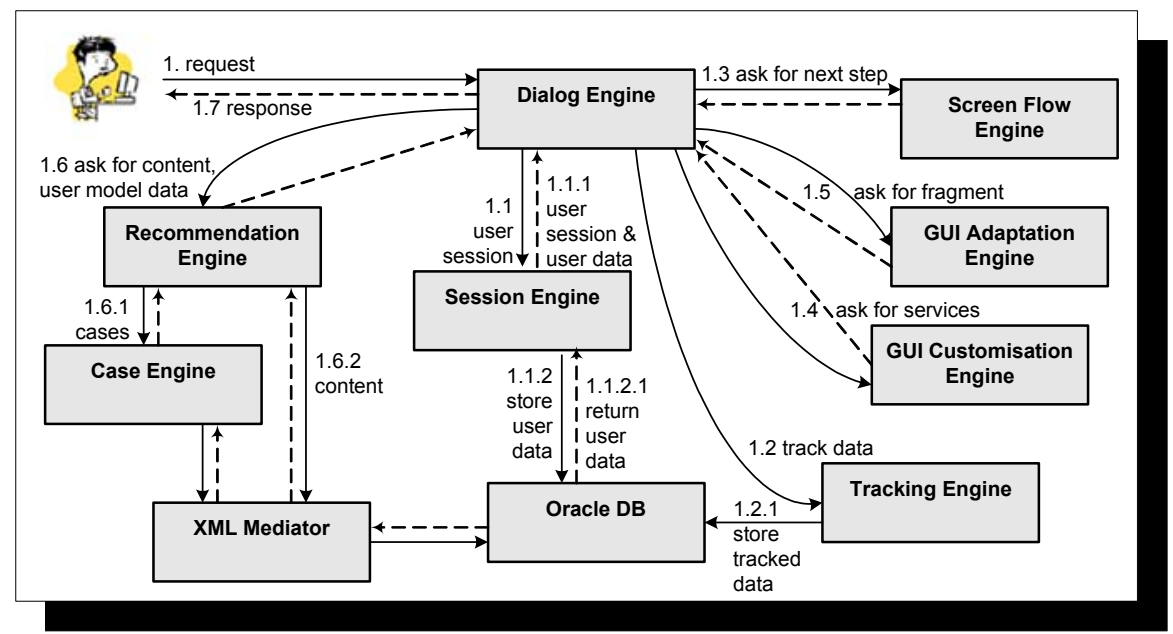

Fig. 2. Cooperation among engines in the Dietorecs system. 
The Dietorecs system is developed as component-based architecture, which consists of several components that interact with each other. The units encapsulate functionalities essential for managing the Dietorecs system and are described in the following, whereby Fig. 2 shows the cooperation among those engines. The Dialog Engine serves as a central management component to support the interaction between user and system, to read the input, to validate it, to manage the workflow and to return the response back to the user. The Session Engine is responsible for authenticating and authorizing users according to their identification and permission rights as well as guaranteeing that a user's session will not be lost during interaction. It overcomes stateless HTTP connections by keeping sessions alive. The Tracking Engine manages tracking of interesting data such as user input or user behaviour. Tracked data is logged in the local database and provides methods to retrieve once tracked and logged data to support the recommendation process.

The Screen Flow Engine informs state-dependent engines about workflow sequences. It is responsible for delivering information about what is the next step that should be delivered to the user. The GUI Customisation Engine provides interaction services independent from content that have to be customised for an individual user. The GUI Adaptation Engine handles all relevant actions for supporting the Dialog Engine with essential layout information for the next required step that has to be visualized and delivered to the user. It assembles a Web page through the adaptation of single fragments described in HTML, XML or JSP.

The Recommendation Engine and Case Engine manage personalized recommendations by considering user-specific travel wish preferences. Together, they are responsible for retrieving ranked travel item or travel bundle recommendations, finding similar travel items or bundles, and adapting travels of previous recommendations to available ones supported by the two tourism information systems APT and Tiscover. The XML Mediator provides access to this data and serves as bridge for Recommendation and Case Engine to get use of this data.

\section{Conclusions}

The proposed Dietorecs system represents a new generation of travel recommender systems that can cope with individual differences in travel wishes and decision styles. We plan to empirically validate the system by $\mathrm{A} / \mathrm{B}$ comparisons with more traditional approaches. The validation will be conducted in Europe (Italy and Austria) and in the US and will provide real time recommendation sessions to be used as training cases for the adaptive behaviour of Dietorecs. 


\section{References}

Crompton, J. L., and P. K. Ankomah. (1993). Choice set propositions in destination decisions. Annals of Tourism Research, 20: 461-476.

Delgado, J. and Davidson, R. (2002). Knowledge bases and user profiling in travel and hospitality recommender systems. In Proceedings of the ENTER 2002 Conference, pages 1-16, Innsbruck, Austria. Springer Verlag.

Fesenmaier, D. R., and J. Jeng. (2000). Assessing structure in the pleasure trip planning process. Tourism Analysis, 5: 13-27.

Grabler, K. and Zins, A.H. (2002) Vacation Trip Decision Styles as Basis for an Automated Recommendation System: Lessons from Observational Studies. In: Wöber, K.W., Frew, A.J. and Hitz, M. (eds.) Information and Communication Technologies in Tourism. Springer: Wien, 458-469.

Hwang, Y.-H., Gretzel, U. and Fesenmaier, D.R. (2002) Behavioural Foundations for HumanCentric Travel Decision-Aid Systems. In: Wöber, K.W., Frew, A.J. and Hitz, M. (eds.) Information and Communication Technologies in Tourism. Springer: Wien, 356-365.

Lue, C. C., J. L. Crompton, and D. R. Fesenmaier. (1993). Conceptualization of multidestination pleasure trip decisions. Annals of Tourism Research, 20: 289-301.

Mayo, E.J. and Jarvis, L.P. (1981) The Psychology of Leisure Travel. Boston: CBI Publishing.

Pazzani, M. J. (1999). A framework for collaborative, content-based and demographic filtering. Artificial Intelligence Review, 13:393-408.

Ricci, F., Arslan, B., Mirzadeh, N. and Venturini, A.(2002a). ITR: a case-based travel advisory system. In Craw, S. and Preece, A. (eds.) 6th European Conference on Case Based Reasoning, ECCBR 2002, Springer: Berlin. 613-627.

Ricci, F., Mirzadeh, N., and Venturini, A. (2002b). Intelligent query managment in a mediator architecture. In 2002 First International IEEE Symposium "Intelligent Systems", pages 221226, Varna, Bulgaria.

Schafer, J. B., Konstan, J. A., and Riedl, J. (2001). E-commerce recommendation applications. Data Mining and Knowledge Discovery, 5(1/2):115-153.

Um, S., and J. L. Crompton. (1990). Attitude determinants in tourism destination choice. Annals of Tourism Research, 17: 432-448.

Woodside, A. G., and S. Lysonski (1989). A general model of traveler destination choice. Journal of Travel Research, 27: 8-14. 